\title{
Catálogo de simbología de apoyo a la representación gráfica del informe técnico de bienes muebles
}
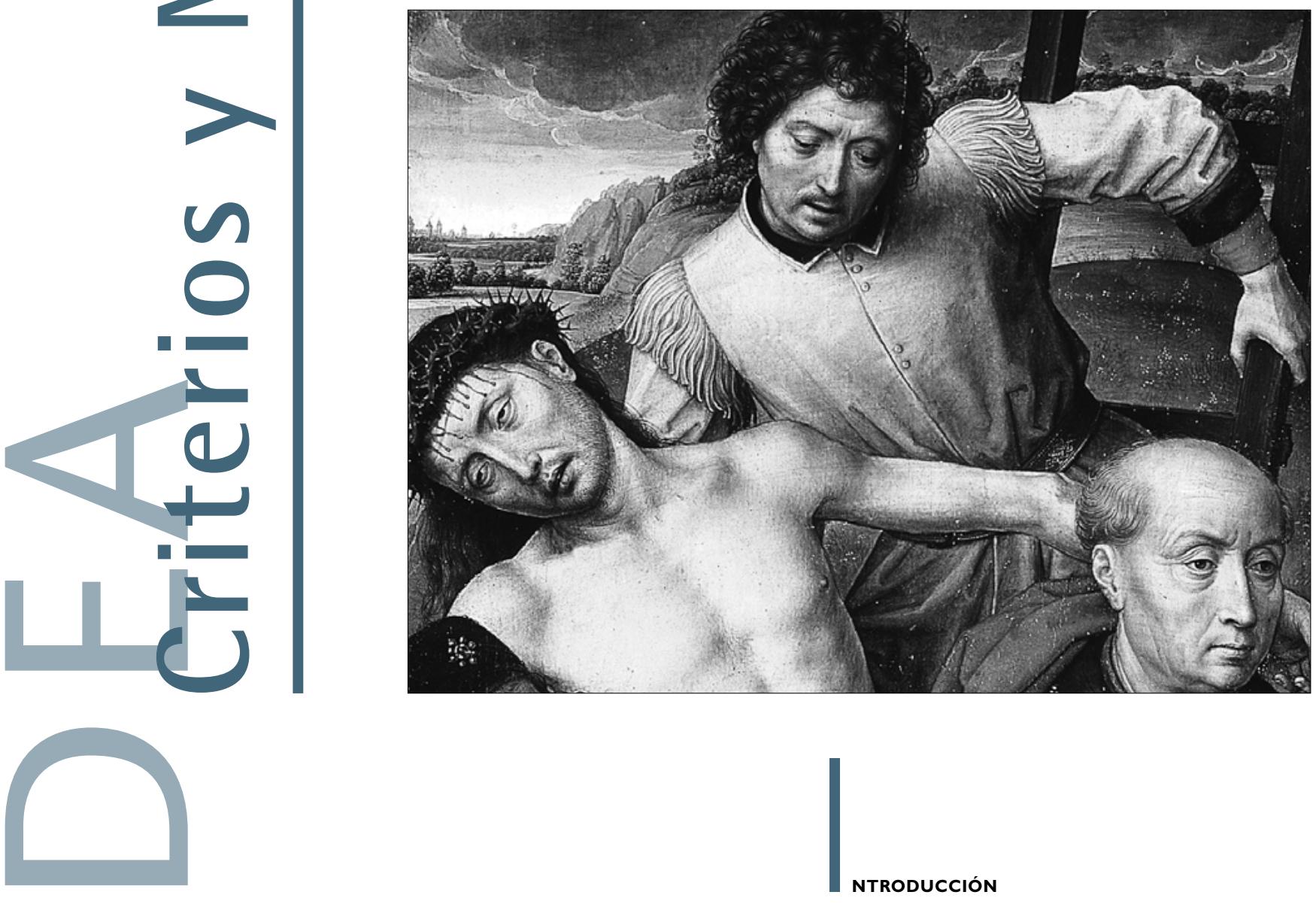

Baglioni, Raniero; González López, $\mathrm{M}^{\mathrm{a}}$ José y López Román, Antonio Centro de Intervención del IAPH
La representación gráfica de un informe técnico tradicionalmente se ha venido empleando con ocasión de la restauración de un bien, y en menor medida, con motivo de su examen. Por lo general, en su realización se ha venido utilizando una simbología aleatoria e individual, en función del técnico que la elabora, y por lo general, sin responder a una metodología predeterminada que homologue el resultado final. En algunas ocasiones, incluso se obvia en el informe y se sustituye por documentación fotográfica de apoyo, que no siempre, es representativa de los que se quiere dejar constancia. Desde nuestra óptica, la representación gráfica es entendida como apoyo de la parte textual 
del informe técnico y como forma de documentar de manera pormenorizada aspectos técnicos, alteraciones, intervenciones anteriores o tratamientos sobre el bien en estudio, en algunas ocasiones no fácilmente registrable con otro método de trabajo, e incluso para facilitar la comprensión de las informaciones desprendidas por parte de personas ajenas a la disciplina de la conservación-restauración.

Desde la creación del Departamento de Tratamiento en el seno del Centro de Intervención del Instituto Andaluz del Patrimonio Histórico (IAPH), nos hemos planteado la inquietud de normalizar tanto la información textual como gráfica que se desprende del informe técnico de un bien mueble, con independencia de la tipología de bienes a la que se aplique. El objetivo de este trabajo ha sido realizar un catálogo de símbolos de apoyo a la representación gráfica de aquellos aspectos representativos, derivados de la investigación, del examen o de la intervención sobre un bien mueble, que responda a la metodología de estudios y actuación puesta a punto para la redacción del informe técnico.

Conceptualmente, y siempre desde la perspectiva del Departamento de Tratamiento de este Instituto, con este catálogo se pretende cumplir una serie de objetivos de régimen interno:

\section{Normalizar la representación gráfica.}

2. Normalizar la terminología empleada.

3. Simplificar la representación gráica mediante la aplicación de una simbología standard.

4. Aplicar el tratamiento informático de los datos al resultado final del informe técnico.

5. Obtener una buena calidad final del trabajo.

6. Facilitar la reproducción de los gráficos resultantes.

7. Homologar el resultado final de la parte gráfica del informe técnico.

Este método de trabajo lo hemos considerado necesario e imprescindible desde el momento en el que se pone en marcha el área de talleres y se intenta homologar las informaciones (textual y gráfica) desprendidas de las intervenciones sobre bien muebles que se realizan en su seno. Los motivos que nos lleva a ello son fácilmente comprensibles si tenemos en cuenta que estas actuaciones se llevan a cabo en el marco de una Institución, como es el IAPH, y que no responden a intervenciones individuales con criterios marcados a discreción de cada técnico que actúa sobre el bien.

Desde esta perspectiva, hemos considerado básico diseñar y definir una serie de instrumentos que facilitase el trabajo del especialista y que a su vez homologase el resultado final de la documentación resultante, en esta caso el informe técnico. La filosofía de este documento "informe técnico" se concibe de forma genérica para cualquier tipo de bien, si bien en cada ficha se pormenoriza las características específicas de cada tipología de bien mueble sobre los que se actúa.

\section{METODOLOGÍA DE TRABAJO}

La elaboración del catálogo que presentamos parte inicialmente de unas fichas gráficas elaboradas por este Departamento para los siguientes bienes muebles: pintura sobre tela, pintura sobre tabla, pintura mural y escultura en madera policromada. Estas fichas están concebidas como complemento gráfico de la parte textual del informe técnico que este Departamento ha elaborado para cada una de estas tipologías.

Esta metodología de trabajo, si bien la estamos aplicando en la actualidad en bienes muebles, puede hacerse extensible a bienes inmuebles con las modificaciones necesarias en la simbología correspondiente.

Las fichas gráficas se han estructurado por cada uno de los estratos constitutivos de los informes puestos a punto en cuatro bloques diferentes, teniendo en cuenta las peculiaridades tipológicas de estos bienes culturales, subdividiéndose en: soporte, preparación/imprimación, dibujo subyacente, capa pictórica y capa de protección.

I er Bloque: Datos técnicos.- Contemplan aspectos derivados de los sistemas constructivos y de las características técnicas de la obra.

$2^{\circ}$ Bloque: Alteraciones.- Comprende aquellas alteraciones naturales o prematuras que presenta el bien en el momento de su estudio o intervención.

3er Bloque: Intervenciones anteriores.- Registran aquella intervenciones que el bien ha podido sufrir durante su historia material.

$4^{\circ}$ Bloque: Tratamiento .- Incluyen aquellos tratamientos realizados durante el curso de la intervención.

Para hacer asequible y fácilmente codificable la representación gráfica de aquellos datos derivados de cada uno de estos cuatro bloques se ha empleado dos métodos diferentes. El primero de ellos parte del diseño individual de aquellos símbolos fácilmente comparables con el contenido que se quiere representar, y en segundo lugar, de la selección de símbolos existentes en tramas comerciales, en este caso mecanormas.

En el diseño de estas fichas se ha tenido en cuenta una serie de consideraciones:

I. Reducir al máximo el número de tramas y de símbolos empleados. Para ello se ha utilizado, siempre que ha sido posible, el mismo símbolo o trama para representar la misma alteración, dato técnico, tratamiento, etc., con independencia del estrato en que se localice, o de la tipología de bien cultural.

2. Emplear el blanco y negro en su elaboración para disminuir los costes de reproducción sin menoscabo de la calidad de la reproducción. 
3. El diseño de la ficha permite en todo momento ampliar su contenido introduciendo fácilmente nuevos símbolos en el momento que se considere necesario.

Una vez elaborada las fichas, definido el catálogo de símbolos y comprobado su idoneidad en los trabajos que hemos desarrollado hasta la fecha en el Departamento, nos faltaba informatizar su contenido, como veremos a continuación.

\section{TRATAMIENTO INFORMÁTICO}

Con objeto de optimizar la eficacia del método expresado anteriormente desarrollado por el Departamento de Tratamiento, desde un punto de vista informático, se ha pensado utilizar una aplicación comercial muy versátil, fácil de manejar y ampliamente difundida: COREL DRAW.

Esta aplicación es una de las más extendidas en el campo del dibujo y del diseño por ordenador. Es un programa muy potente y con múltiples utilidades comercializadas, y que junto con la importante bibliografía que existe sobre Corel Draw, hacen de este programa el ideal para que se manipulen gráficos y esquemas, incluso para la gente que tiene poca experiencia en el campo del dibujo e ilustración en Windows.

Independientemente de la utilización de este programa para dar soporte informático al catálogo de tramas confeccionado, también se puede realizar lo mismo en otros programas comerciales o de libre difusión de dibujo o de tratamiento de imagen por ordenador, con resultados prácticamente similares si se realiza el trabajo adecuadamente.
La trama final se obtiene de la repetición sucesiva de un motivo (icono) que es la base de la misma. En este artículo hemos presentado una colección de tramas muy simples con objeto de que este sistema consuma pocos recursos físicos del ordenador, es decir, que se pueda utilizar en ordenadores modestos.

Aunque el programa que estamos utilizando presenta múltiples opciones, todas muy interesantes, nosotros nos vamos a centrar exclusivamente en la aplicación directa de las tramas que hemos diseñado sobre el método de trabajo anteriormente expuesto, dejando de lado otras utilidades del programa ya que no son objeto de este artículo.

Cada una de las zonas tramadas se corresponden con una característica, alteración o intervención del bien en estudio. Es evidente que para poder perfilar las zonas de interés e implementarla en el gráfico correspondiente, hay que digitalizar el esquema de la obra en la que se trabaje y posteriormente con las herramientas adecuadas definir cual es la zona a rellenar.

En la figura I (opción a y b) se expone el procedimiento para llegar a la utilidad donde están los iconos que constituyen las tramas. El icono sólo hay que seleccionarlo y aplicarlo a la zona que se desee. En la opción c de la figura I, se muestra una elipse vacía de contenido, y en la opción d, la misma elipse después de ser tratada con el motivo de la trama elegida, en este caso un círculo.

Aunque el sistema es bastante fácil de manejar se pueden presentar los siguientes problemas:

I. Sólo se podrán rellenar áreas cerradas. Hay que asegurarse que las áreas que delimitan nuestra zona

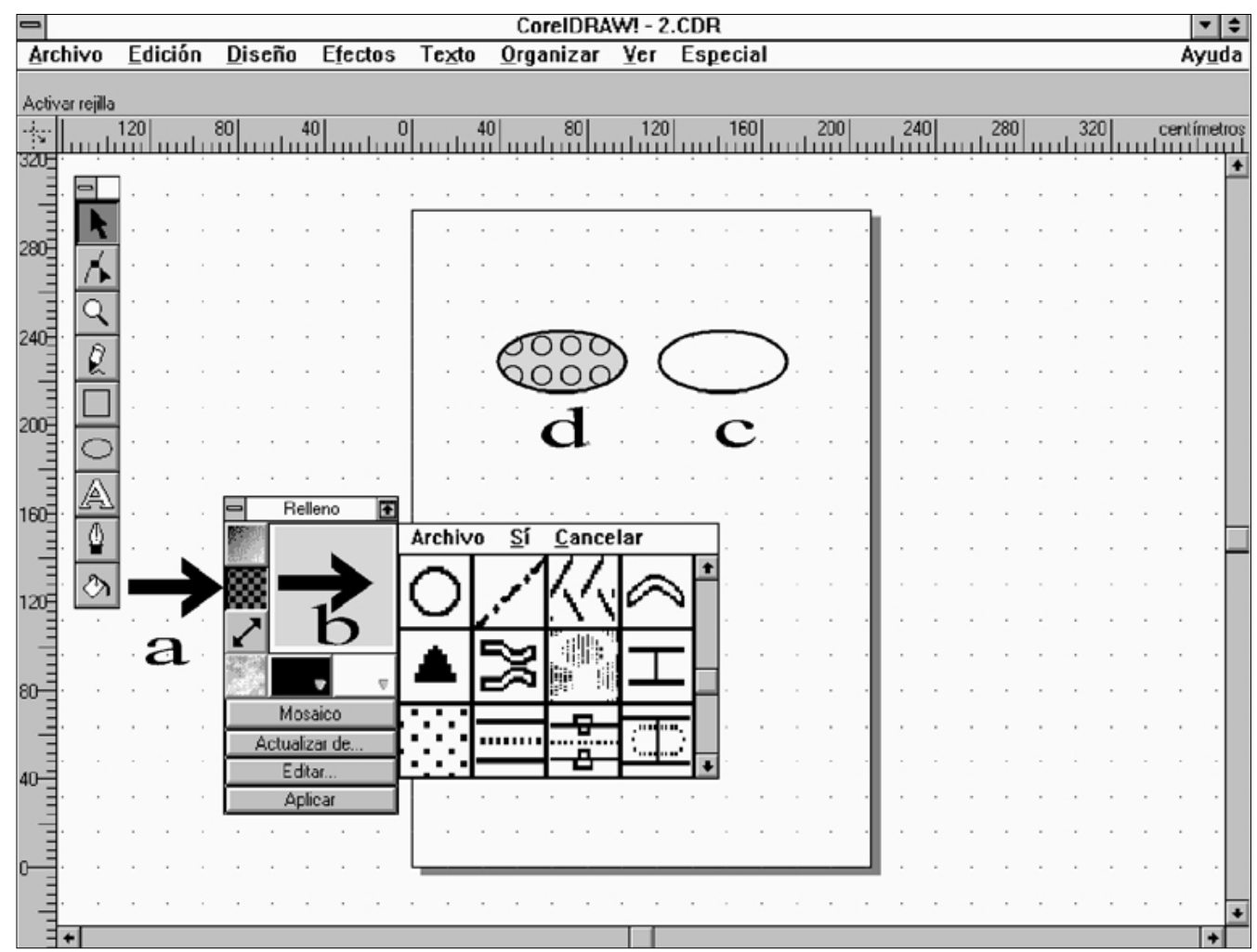


de interés están cerradas. En la figura 2 se muestra un ejemplo de un área cerrada donde se puede utilizar una trama.

2. La zona rellena de tramas queda encima del esquema tapando las líneas que lo forma. Esto se resuelve seleccionando la zona tramada y en el menú principal elegir la opción organizar, dentro de esta orden y seleccionando hacia atrás. De esta forma la zona tramada no tapa la imagen original. En la figura 2 se muestra el proceso completo de izquierda a derecha.

3. Si el dibujo de base (la imagen femenina que se observa en la figura 2) se ha realizado en escala de grises o de colores, aunque sólo se utilice el blanco y el negro, la trama no se podrá ver a través del dibujo. Es imprescindible para evitar este problema y obtener la transparencia deseada, que el tipo de datos que conforme el dibujo base sea de I bit (blanco y negro) o imagen de líneas.

\section{ESTRUCTURA DEL CATÁLOGO:}

El catálogo de símbolos parte de los modelos de fichas gráficas diseñadas para cada una de las tipologías de bienes culturales puesta a punto: pintura (tela, tabla y pintura mural) y escultura en madera policromada. Su estructura se realiza en dos bloques diferentes, el primero de ellos denominado "genérico" y, el segundo, denominado "específico".

En la parte genérica del catálogo se incluyen aquellos símbolos generales aplicables a todas las tipologías estudiadas. Véase tabla $n^{\circ}$ I.
En la parte especíica del catálogo se contempla aquella simbología particular de cada tipología de bienes que presentamos en este artículo, Véase tabla $n^{\circ} 2$.

En ambos casos, se parte de una estructura que intenta representar información de cada uno de los estratos constitutivos que pueden configurar estos bienes culturales, según se contempla en las tablas $n^{\circ} \mid$ y $n^{\circ} 2$

A fin de facilitar la búsqueda del símbolo relacionado con el dato que se quiere representar se ha establecido un código de búsqueda estructurado de la siguiente forma:

Bloque genérico: Configurado por dos dígitos, que se corresponden con letras. El primer dígito representa la información (datos técnico, alteración, intervenciones anteriores o tratamiento) y el segundo, su localización (estrato).

Bloque específico: Compuesto por tres dígitos, constituidos por letras. El primero de ellos corresponde con la tipología, el segundo con la información y el tercero, con el estrato.

\section{CONCLUSIONES}

Con este trabajo hemos querido poner en conocimiento de un público especializado la metodología de representación gráfica que este Departamento ha venido empleando en la realización de los informes técnicos de cada una de las intervenciones de conservación-restauración que ha venido efectuando desde su creación en 1990. La experiencia de los trabajos realizados avalan la validez del método y ha puesto de manifiesto el alcance de los objetivos prefijados de ante-

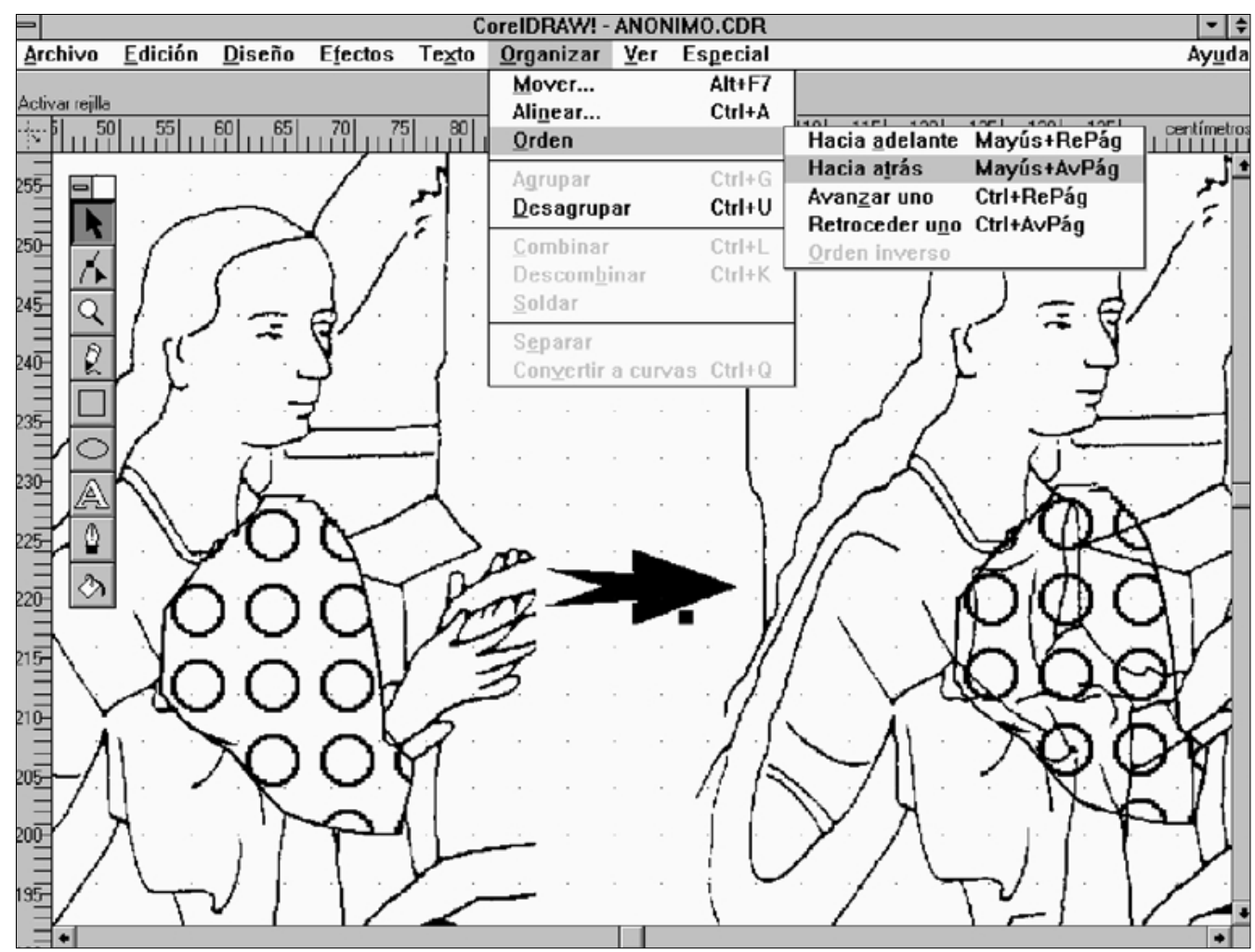


mano, desde el punto de vista, que se ha conseguido uniformar y homologar las informaciones gráficas contenidas en cada uno de los informes elaborados.

En ningún caso pretendemos que sea entendido como una propuesta de normalización, sino como divulgación de una metodología de representación gráfica que puede ser fácilmente utilizada por cualquier especialista o Institución que considere de interés su implementación en su método de trabajo.
Es por ello, que no sólo ponemos a disposición del público el catálogo de símbolos, sino también, el fichero gráfico donde están recogidas cada una de las tramas en el contenida, que podrá ser solicitado a este Instituto a partir de este momento, ya que se incluye como un servicio más de los que este Instituto puede hoy día ofrecer, como queda contemplado en su Catálogo de Servicios.

Tabla $n^{\circ}$ I. ESTRUCTURA DEL CATÁLOGO: BLOQUE GENÉRICO

\begin{tabular}{llc}
\hline INFORMACIÓN & EXTRATO & CÓDIGO \\
\hline DATOS TÉCNICOS (T) & SOPORTE (S) & TS \\
& PREPARACIÓN/IMPRIMACIÓN (P) & TP \\
& DIBUJO SUBYACENTE (D) & TD \\
& CAPA DE COLOR (C) & TC \\
\hline ALTERACIONES (A) & CAPA DE PROTECCIÓN (B) & TB \\
& SOPORTE (S) & AS \\
& PREPARACIÓN/IMPRIMACIÓN (P) & AP \\
& DIBUJO SUBYACENTE (D) & AD \\
INTERVENCIONES ANTERIORES (I) & CAPA DE COLOR (C) & $\mathrm{AC}$ \\
& CAPA DE PROTECCIÓN (B) & $\mathrm{AB}$ \\
\hline TRATAMIENTO (R) & SOPORTE (S) & $\mathrm{IS}$ \\
& PREPARACIÓN/IMPRIMACIÓN (P) & $\mathrm{IP}$ \\
& DIBUJO SUBYACENTE (D) & $\mathrm{ID}$ \\
& CAPA DE COLOR (C) & IC \\
& CAPA DE PROTECCIÓN (B) & $\mathrm{IB}$ \\
\hline & SOPORTE (S) & $\mathrm{RS}$ \\
& PREPARACIÓN/IMPRIMACIÓN (P) & $\mathrm{RP}$ \\
& DIBUJO SUBYACENTE (D) & $\mathrm{RD}$ \\
& CAPA DE COLOR (C) & $\mathrm{RC}$ \\
& CAPA DE PROTECCIÓN (B) & $\mathrm{RB}$
\end{tabular}

Tabla $n^{\circ}$ 2. ESTRUCTURA DEL CATÁLOGO: BLOQUE ESPECÍFICO

\begin{tabular}{|c|c|c|c|}
\hline TIPOLOGÍA & INFORMACIÓN & ESTRATO & CÓDIGO \\
\hline $\begin{array}{l}\text { PINTURA TELA (L)) } \\
\text { PINTURA TABLA ( F) } \\
\text { PINTURA MURAL (U) } \\
\text { ESCULTURA EN MADERA (M ) }\end{array}$ & DATOS TÉCNICOS (T) & $\begin{array}{l}\text { SOPORTE (S) } \\
\text { PREPARACIÓN/IMPRIMACIÓN (P) } \\
\text { DIBUJO SUBYACENTE (D) } \\
\text { CAPA DE COLOR (C) } \\
\text { CAPA DE PROTECCIÓN (B) }\end{array}$ & $\begin{array}{l}\text { LTS. FTS, UTS, MTS } \\
\text { LTP, FTP, UTP, MTP } \\
\text { LTD, FTD, UTD, MTD } \\
\text { LTC ,FTC, UTC, MTC } \\
\text { LTB, FTB, UTB, MTB }\end{array}$ \\
\hline $\begin{array}{l}\text { PINTURA TELA (L)) } \\
\text { PINTURA TABLA ( F) } \\
\text { PINTURA MURAL (U) } \\
\text { ESCULTURA EN MADERA (M ) }\end{array}$ & ALTERACIONES (A) & $\begin{array}{l}\text { SOPORTE (S) } \\
\text { PREPARACIÓN/IMPRIMACIÓN (P) } \\
\text { DIBUJO SUBYACENTE (D) } \\
\text { CAPA DE COLOR (C) } \\
\text { CAPA DE PROTECCIÓN (B) }\end{array}$ & $\begin{array}{l}\text { LAS. FAS, UAS, MAS } \\
\text { LAP, FAP, UAP, MAP } \\
\text { LAD, FAD, UAD, MAD } \\
\text { LAC, FAC, UAC, MAC } \\
\text { LAB, FAB, UAB, MAB }\end{array}$ \\
\hline $\begin{array}{l}\text { PINTURA TELA (L)) } \\
\text { PINTURA TABLA ( F) } \\
\text { PINTURA MURAL (U) } \\
\text { ESCULTURA EN MADERA (M ) }\end{array}$ & INTERVENCIONES ANTERIORES (I) & $\begin{array}{l}\text { SOPORTE (S) } \\
\text { PREPARACION/IMPRIMACIÓN (P) } \\
\text { DIBUJO SUBYACENTE (D) } \\
\text { CAPA DE COLOR (C) } \\
\text { CAPA DE PROTECCIÓN (B) }\end{array}$ & $\begin{array}{l}\text { LIS. FIS, UIS, MIS } \\
\text { LIP, FIP, UIP, MIP } \\
\text { LID, FID, UID, MID } \\
\text { LIC ,FIC, UIC, MIC } \\
\text { LIB, FIB, UIB, MIB }\end{array}$ \\
\hline $\begin{array}{l}\text { PINTURA TELA (L)) } \\
\text { PINTURA TABLA ( F) } \\
\text { PINTURA MURAL (U) } \\
\text { ESCULTURA EN MADERA (M ) }\end{array}$ & TRATAMIENTO (R) & $\begin{array}{l}\text { SOPORTE (S) } \\
\text { PREPARACIÓN/IMPRIMACIÓN (P) } \\
\text { DIBUJO SUBYACENTE (D) } \\
\text { CAPA DE COLOR (C) } \\
\text { CAPA DE PROTECCIÓN (B) }\end{array}$ & $\begin{array}{l}\text { LRS. FRS, URS, MRS } \\
\text { LRP, FRP, URP, MRP } \\
\text { LRD, FRD, URD, MRD } \\
\text { LRC, FRC, URC, MRC } \\
\text { LRB, FRB, URB, MRB }\end{array}$ \\
\hline
\end{tabular}




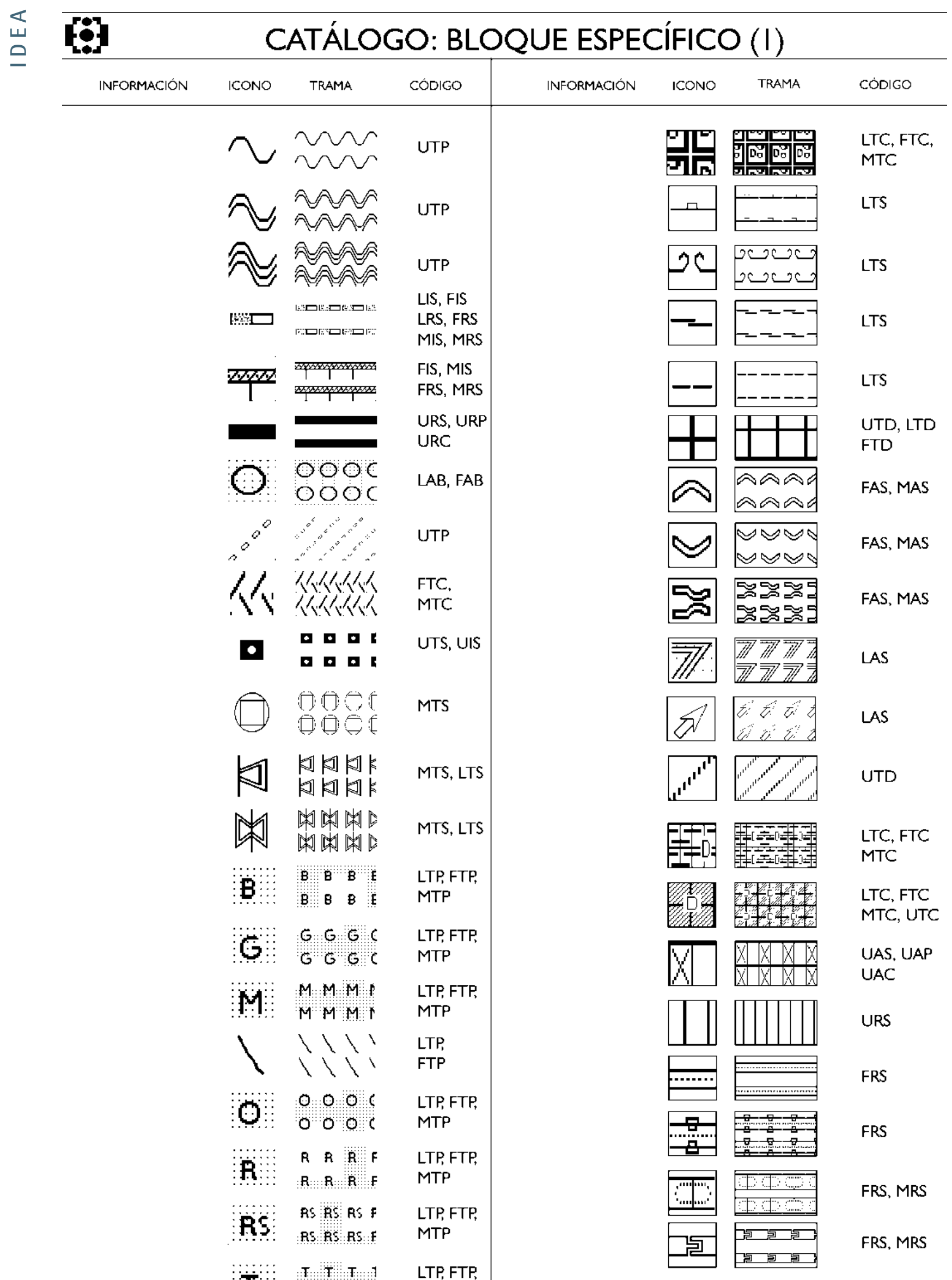


6 CATÁLOGO: BLOQUE ESPECÍFICO (2)

\begin{tabular}{|c|c|c|c|c|c|c|c|}
\hline INFORMACIÓN & ICONO & TRAMA & CóD|GO & INFORMACIÓN & KONO & TRAMA & CóDIGO \\
\hline & & 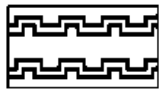 & FRS, MRS & & (1) & $\begin{array}{ll}11 \\
1\end{array}$ & UTS \\
\hline & & 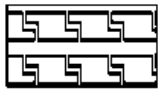 & FRS, MRS & & & 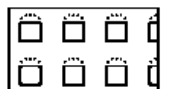 & $\begin{array}{l}\text { LIS, FIS } \\
\text { MIS }\end{array}$ \\
\hline & & & MRS & & 田 & $\begin{array}{l}\theta \\
\nabla\end{array}$ & FTS, MTS \\
\hline & & $\begin{array}{lll} & 1\end{array}$ & FRS, MRS & & & W4 & UTP \\
\hline & & & FRS, MRS & & (9) & (3) 의의 & FTS, MTS \\
\hline & & & $\begin{array}{l}\text { LTC, FTC } \\
\text { MTC }\end{array}$ & & & 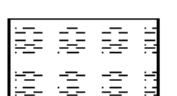 & LIS, LRS \\
\hline & & $\begin{array}{l}2 \\
P\end{array}$ & FAS, MAS & & & & UTC \\
\hline & & & UTP & & 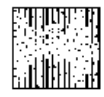 & 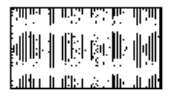 & $\begin{array}{l}\text { LAB, FAB } \\
\text { MAB }\end{array}$ \\
\hline & & & $\begin{array}{l}\text { LAS, FAS } \\
\text { MAS }\end{array}$ & & $\mathrm{P}$ & 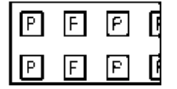 & MTS \\
\hline & & $\begin{array}{l}\square \square \square \\
\square \square \square \square\end{array}$ & UTS & & & $\begin{array}{c}\triangle A \\
\wedge \wedge\end{array}$ & $\begin{array}{l}\text { UIS, UIP } \\
\text { UIC }\end{array}$ \\
\hline & & & $\begin{array}{l}\text { FTS, UTS } \\
\text { MTS }\end{array}$ & & & 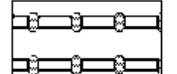 & UTS \\
\hline & & 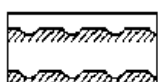 & UTP & & & 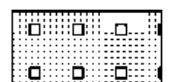 & MTC \\
\hline & 60 & $\begin{array}{l}8000080 \\
3000000 \\
0000000 \\
000000\end{array}$ & FAS, MAS & & 口 & $\begin{array}{|lll|}0 & 0 & 0 \\
0 & 0 & 0 \\
\end{array}$ & MTC \\
\hline & 曲 & 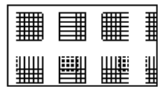 & LRS & & & 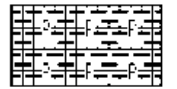 & $\begin{array}{l}\text { LTC, FTC } \\
\text { MTC }\end{array}$ \\
\hline & & & UTP & & & & UTP \\
\hline & & |ח & UTS & & & E-ニ- & $\begin{array}{l}\text { LTP, FTP } \\
\text { MTP }\end{array}$ \\
\hline & & 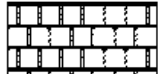 & UTS & & & al & $\begin{array}{l}\text { LTP, FTP } \\
\text { MTP }\end{array}$ \\
\hline & & 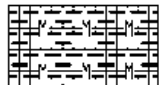 & $\begin{array}{l}\text { LTC, FTC } \\
\text { MTC }\end{array}$ & & & & UTP \\
\hline & & & UAS & & - & $\begin{array}{lll}1 & . & 1 \\
. & . & 1\end{array}$ & UTP \\
\hline & & mints & UTS & & $\because \cdots$ & b๐ & FTP \\
\hline & & חדיבדים & UTS & & WIII) & 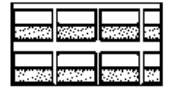 & FIP \\
\hline
\end{tabular}




\begin{tabular}{|c|c|c|c|c|}
\hline 6 & \multicolumn{4}{|c|}{ CATÁLOGO: BLOQUE ESPECIFFCO (3) } \\
\hline & & 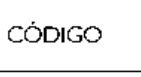 & & $\cos _{0 \rightarrow \infty}$ \\
\hline & 工曲糕 & us & 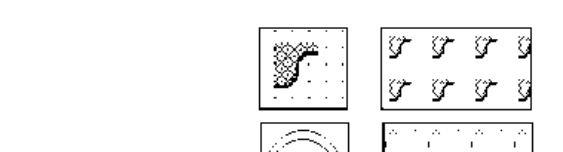 & 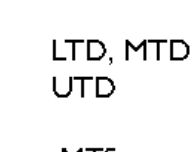 \\
\hline & 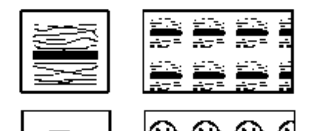 & us & $0 \quad 5$ & \\
\hline & 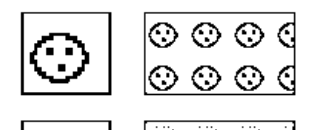 & res mins & & mis \\
\hline & 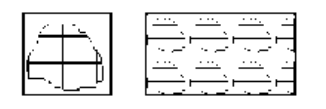 & urs & mer & Frs UTS \\
\hline & & uare & 브閶間 & uss \\
\hline & 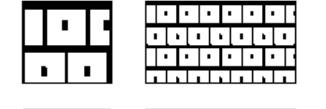 & FES MAS & 可㗊器 & Fis M.MES \\
\hline & $\square$ & urs & 굴 & 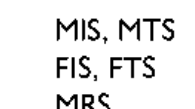 \\
\hline & 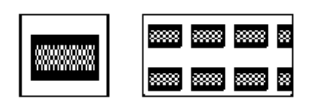 & us & 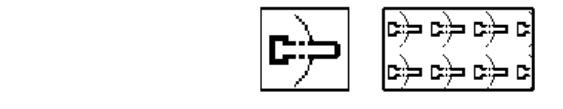 & ms \\
\hline & 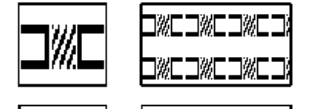 & 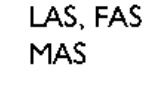 & 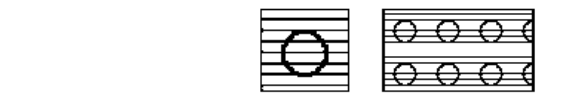 & ThAP \\
\hline & 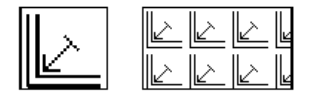 & ths & 男 & Fis \\
\hline
\end{tabular}

\section{CATÁLOGO: BLOQUE GENÉRICO (I)}

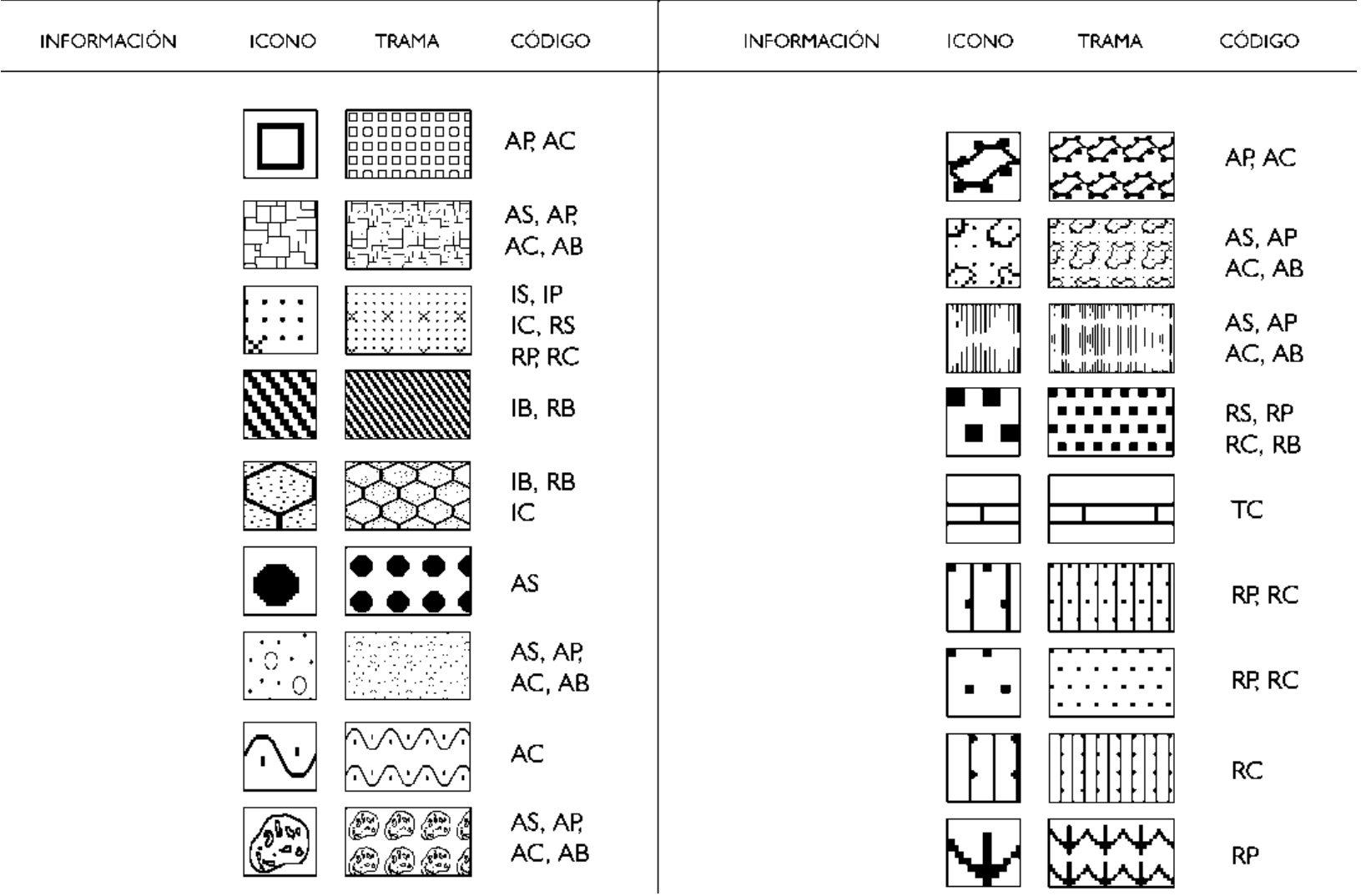


6 CATÁLOGO: BLOQUE GENÉRICO (2)

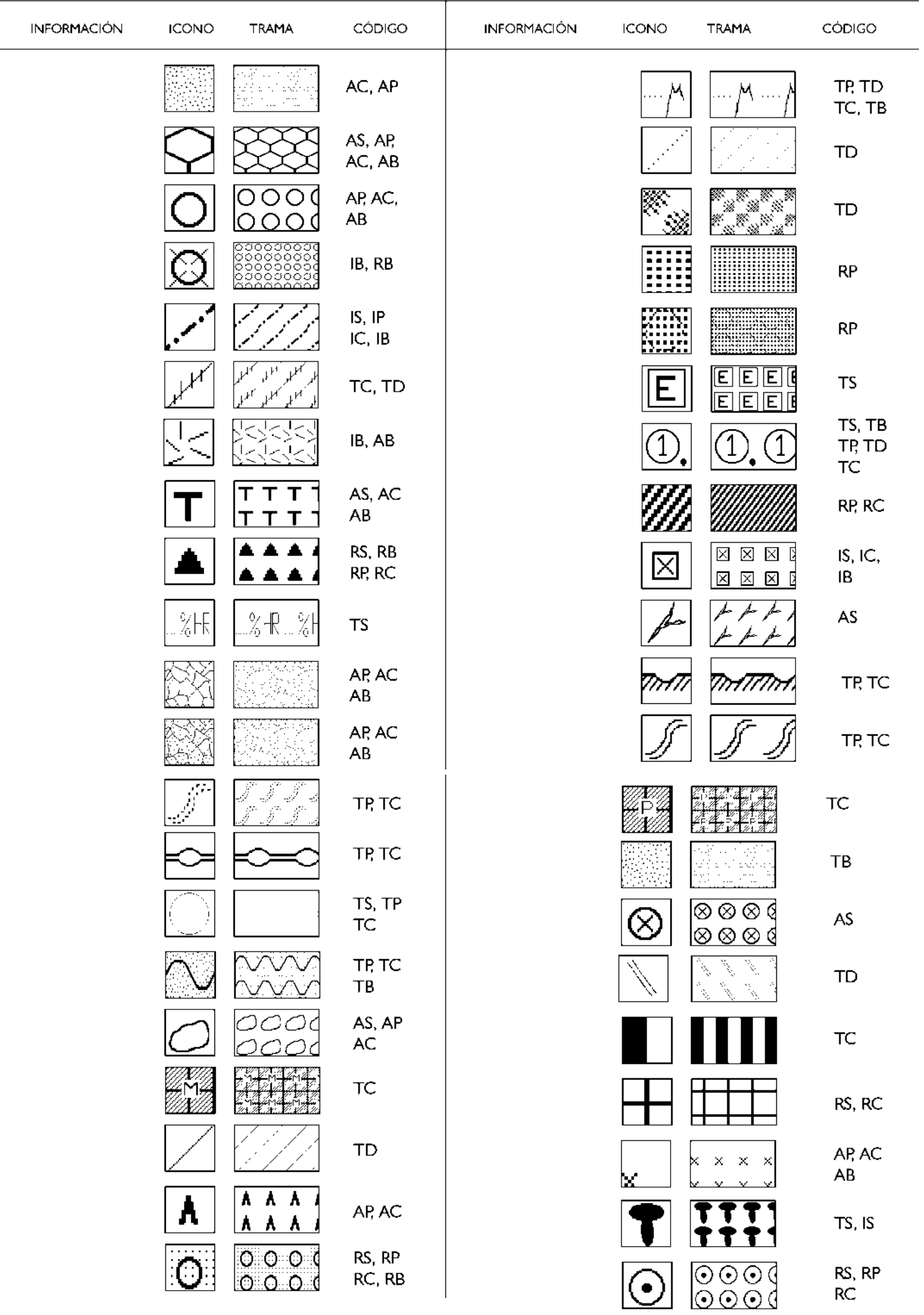




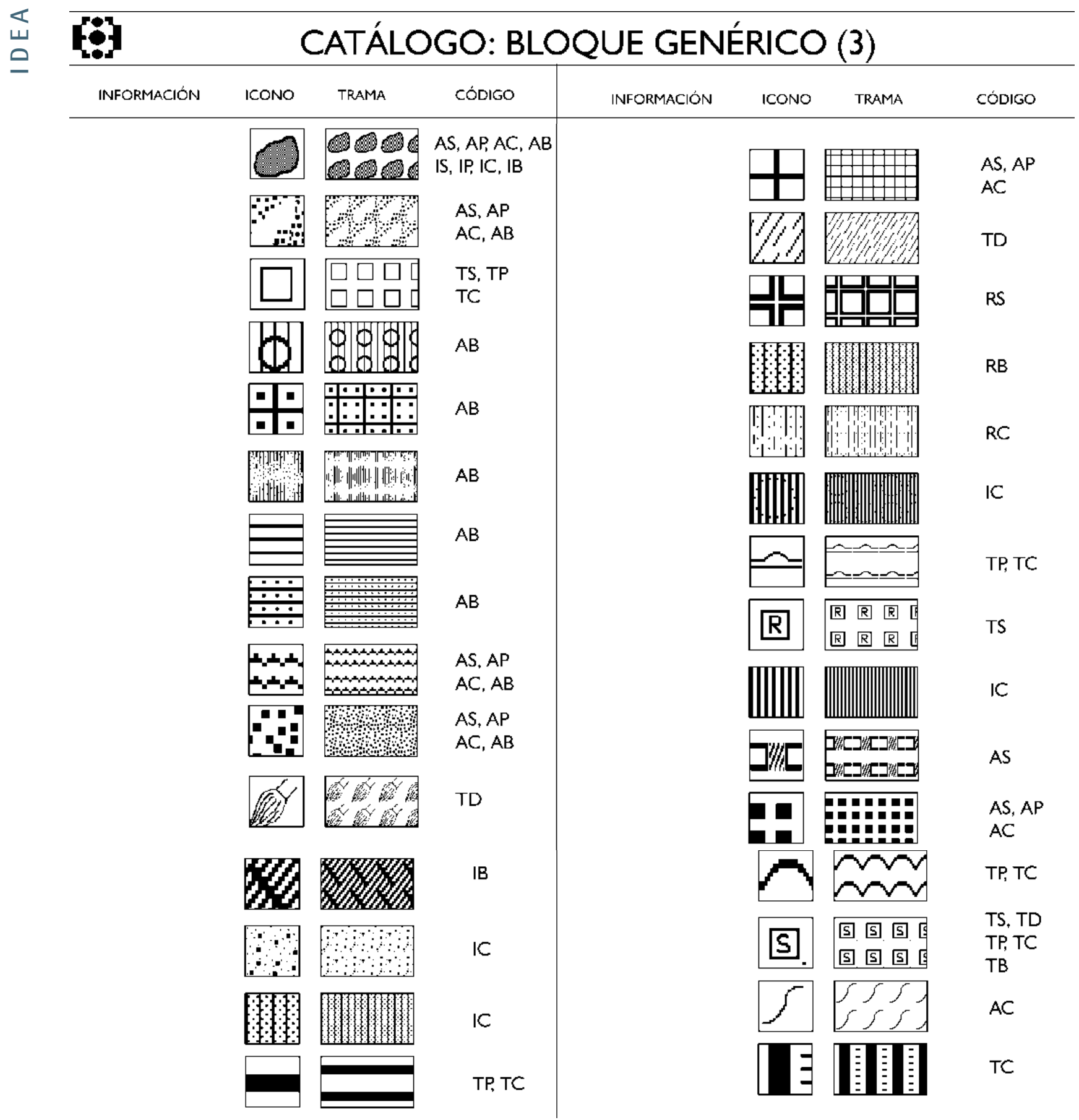

Ficha técnica

Puesta a punto del método de trabajo y diseño del catálogo:

Baglioni, Raniero

Sector Conservación Preventiva del Centro de Intervención del IAPH.

González López, Mํasé

Jefe del Departamento de Tratamiento del Centro de Intervención del IAPH.
Colaboradores:

Magdaleno Granja, Rocío

Moreno Montero, Araceli.

Departamento de Tratamiento del IAPH.

Tratamiento informático:

López Román, Antonio

Departamento de Análisis del Centro de Intervención del IAPH. 